\title{
All-Fiber DBR-Based Sensor Interrogation System for Measuring Acoustic Waves
}

\author{
Maria Iulia Comanici, ${ }^{1}$ Lv Zhang, ${ }^{1}$ Lawrence R. Chen, ${ }^{1}$ Xijia Gu, ${ }^{2}$ \\ Lutang Wang, ${ }^{3}$ and Peter Kung ${ }^{3}$ \\ ${ }^{1}$ Department of Electrical and Computer Engineering, McGill University, Montreal, QC, Canada H3A $2 A 7$ \\ ${ }^{2}$ Department of Electrical and Computer Engineering, Ryerson University, Toronto, ON, Canada M5B $2 \mathrm{~K} 3$ \\ ${ }^{3}$ QPS Photronics, Pointe Claire, QC, Canada H9R 5L7
}

Correspondence should be addressed to Lawrence R. Chen, lawrence.chen@mcgill.ca

Received 2 December 2011; Accepted 17 February 2012

Academic Editor: Wolfgang Ecke

Copyright (C) 2012 Maria Iulia Comanici et al. This is an open access article distributed under the Creative Commons Attribution License, which permits unrestricted use, distribution, and reproduction in any medium, provided the original work is properly cited.

\begin{abstract}
We investigate the use of all-fiber distributed Bragg reflector (DBR) lasers for fiber optic sensing. We measure the steady-state strain response and show that it is very similar to that for a simple fiber Bragg grating (FBG). The lasers can be wavelength multiplexed and support multisensor operation without crosstalk. We also verify the principle of wavelength-to-power mapping, which can simplify sensor interrogation. Finally, we demonstrate that all-fiber DBR lasers can be used to detect acoustic waves.
\end{abstract}

\section{Introduction}

The fiber optic nature of fiber Bragg gratings (FBGs) makes them useful as sensing elements because they are compact, low cost, and immune to electromagnetic interference. Environmental and mechanical factors affect the response of FBGs: in particular, their reflection spectra shift to different wavelengths in response to changes in temperature, applied strain, or pressure $[1,2]$.

The wavelength-encoded nature of the information associated with FBG sensors associated with FBG sensors allows them to be wavelength multiplexed, that is, WDM configurations. Sensor interrogation and information recovery requires the use of an optical spectrum analyzer (OSA) to measure accurately the changes in wavelength [3]. On the other hand, the ability to map wavelength-encoded information to power measurements can simplify system design and reduce cost; indeed, various implementations of wavelength-to-power mapping for FBG sensors have been demonstrated using interrogation filters such as edge filters and arrayed-waveguide gratings (AWGs) [4-10]. This interrogation method allows using power meters or photodetectors to monitor the detected signal from the sensor.
However, such systems suffer from low power efficiency, that is, the signal reflected by an FBG sensor is typically low in power due to the spectral slicing process, thereby resulting in a limited dynamic range and decreased sensitivity. While it has been shown that the resolution of the wavelength-topower mapping can be improved with FBGs having narrow bandwidth [10], this exacerbates the problem of power efficiency as even less power is reflected by the sensor.

Recently, we proposed a simple change to improve the overall performance of the conventional FBG-interrogation filter system: we use optical feedback to create a linear cavity laser [11]. With this laser configuration, there is increased output power at the FBG sensor wavelengths due to lasing, which results in a greater dynamic range and higher sensitivity as it is easier to distinguish the lasing peak from the noise floor. Moreover, the bandwidth of the signal reflected by the FBG sensor is reduced (since the lasing linewidth is narrower than the FBG reflection bandwidth), which improves the system resolution. Although the laser system works well for steady-state strain/temperature measurements, crosstalk in the laser gain medium, either a semiconductor optical amplifier or an Erbium-doped fiber amplifier (EDFA), prohibits its use for dynamic measurements [12]. 


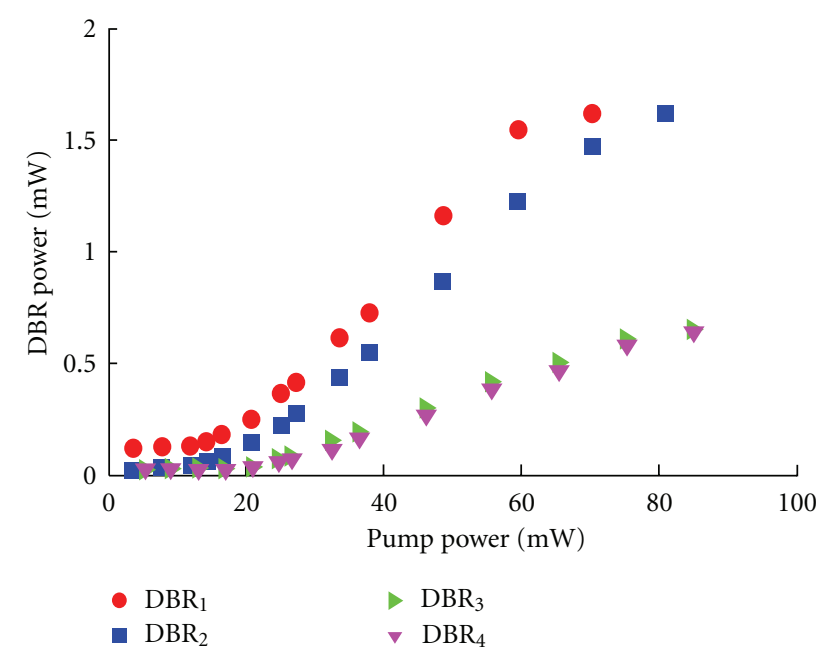

Figure 1: Output power from DBR lasers as a function of pump power.

TABLE 1: Threshold pump power for the DBRs used in the experiment.

\begin{tabular}{lc}
\hline & Threshold pump power $(\mathrm{mW})$ \\
\hline $\mathrm{DBR}_{1}$ & 13.13 \\
$\mathrm{DBR}_{2}$ & 15.21 \\
$\mathrm{DBR}_{3}$ & 16.88 \\
$\mathrm{DBR}_{4}$ & 19.49 \\
\hline
\end{tabular}

In this paper, we investigate all-fiber distributed Bragg reflector (DBR) lasers for fiber optic sensing. We show that the lasing wavelengths shift with applied strain with sensitivities similar to conventional FBG sensors. Since the lasers are spatially separate and each has its own gain medium, gain competition and the resulting deleterious effects of crosstalk can be minimized, if not avoided. Finally, we demonstrate that the DBR sensors can be used to detect and locate acoustic waves.

\section{Experimental Setup and All-Fiber DBR Sensor Characterization}

Each DBR laser used in our experiment comprises two FBGs at the same wavelength $(\sim 1533 \mathrm{~nm})$ and separated by approximately $8 \mathrm{~mm}$ of Er:Yb codoped fiber (with an absorption of $2 \mathrm{~dB} / \mathrm{cm}$ at $980 \mathrm{~nm}$ ). The FBGs are written using UV exposure, and the Er:Yb codoped fiber is hydrogen loaded to increase its photosensitive response. One FBG has high reflectivity $(\sim 99 \%)$ and is broadband, covering several longitudinal modes, while the second FBG has lower reflectivity (but $>90 \%$ ) and spans only one or two longitudinal modes. Asymmetry in the gain profile typically causes the oscillation of only one longitudinal mode. Figure 1 shows the measured output power versus pump power for the four DBR lasers used; the threshold powers are given in Table 1 .

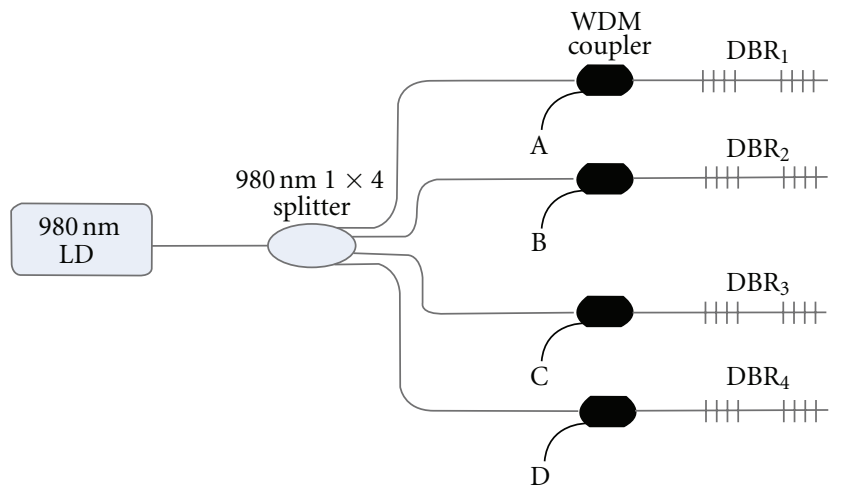

(a)

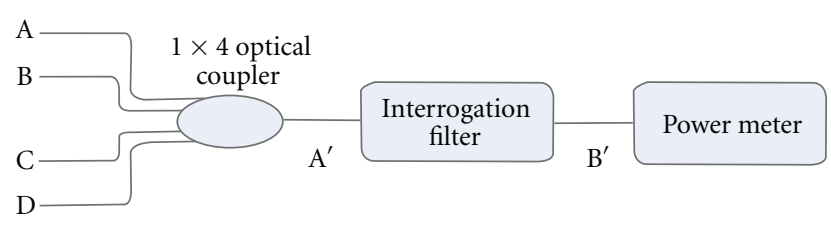

(b)

Figure 2: (a) Experimental setup of 4 DBR sensor system and (b) demodulation system used to measure the response of each sensor. LD: laser diode.

The experimental setup for a four DBR sensor system is shown in Figure 2. The output from the $980 \mathrm{~nm}$ pump laser (maximum output of $360 \mathrm{~mW}$ ) is distributed equally among 4 branches and launched to the DBR sensors using 980/1550 WDM couplers. The output signals from each DBR sensor are coupled and then an arrayed waveguide grating (AWG) or tunable bandpass filter is used for wavelengthto-power mapping. Figure 3(a) shows the optical spectrum of the combined signals at port $\mathrm{A}^{\prime}$; nearly equal power is available from the four DBR sensors. Note that since the four DBRs operate nominally at the same wavelength, we placed them on separate translation stages to shift their wavelength for easier identification. Figure 3(b) shows the typical optical spectrum for a single DBR laser $\left(\mathrm{DBR}_{4}\right)$; the optical signal to noise ratio is $58 \mathrm{~dB}$.

We first characterize the strain response of each DBR sensor. Figure 4(a) shows the measured wavelength shift as a function of applied strain. We obtain strain sensitivities of $0.85 \mathrm{~nm} / \mathrm{mstrain}, 0.88 \mathrm{~nm} / \mathrm{mstrain}, 0.86 \mathrm{~nm} / \mathrm{mstrain}$, and $0.84 \mathrm{~nm} / \mathrm{mstrain}$ for $\mathrm{DBR}_{1}, \mathrm{DBR}_{2}, \mathrm{DBR}_{3}$, and $\mathrm{DBR}_{4}$, respectively. These values are similar to the typical FBG strain sensitivity of $1 \mathrm{~nm} / \mathrm{mstrain}$ [13]. Next, the DBR signals are coupled and sent to an AWG for wavelengthto-power mapping. The output from each AWG channel is detected using a $125 \mathrm{MHz}$ photodetector followed by a $2 \mathrm{MHz}$ electrical low pass filter. The wavelength of each DBR was tuned over the wavelength span of the corresponding AWG channel and the detected voltage as a function of applied strain was obtained; the result for $\mathrm{DBR}_{4}$ is illustrated in Figure 4(b). The detected voltage follows the shape of the AWG channel spectral response, thereby verifying successful wavelength-to-power mapping. 


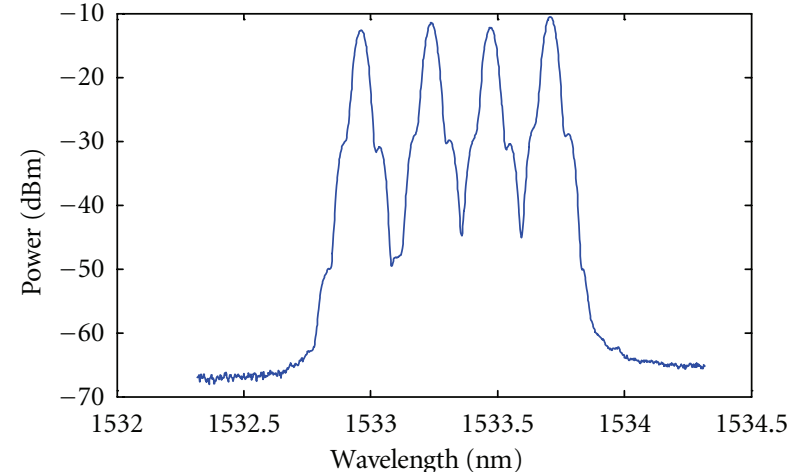

(a)

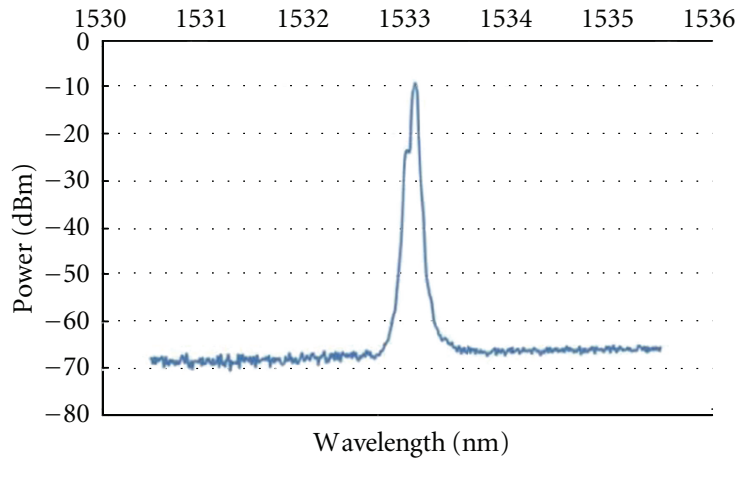

(b)

FIgURE 3: (a) Optical spectrum measured at point $\mathrm{A}^{\prime}$ showing equal power from the $4 \mathrm{DBR}$ sensors. (b) Output spectrum from $\mathrm{DBR}_{4}$.

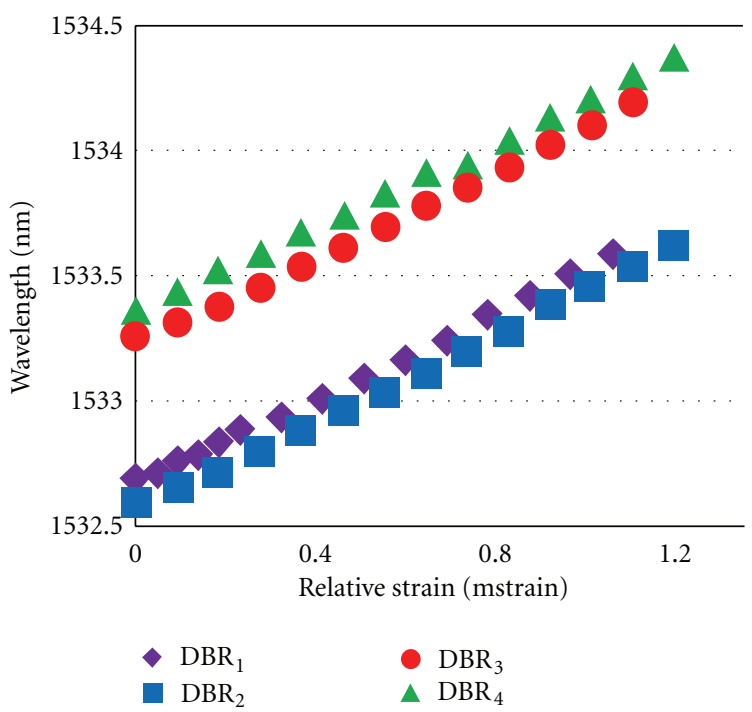

(a)

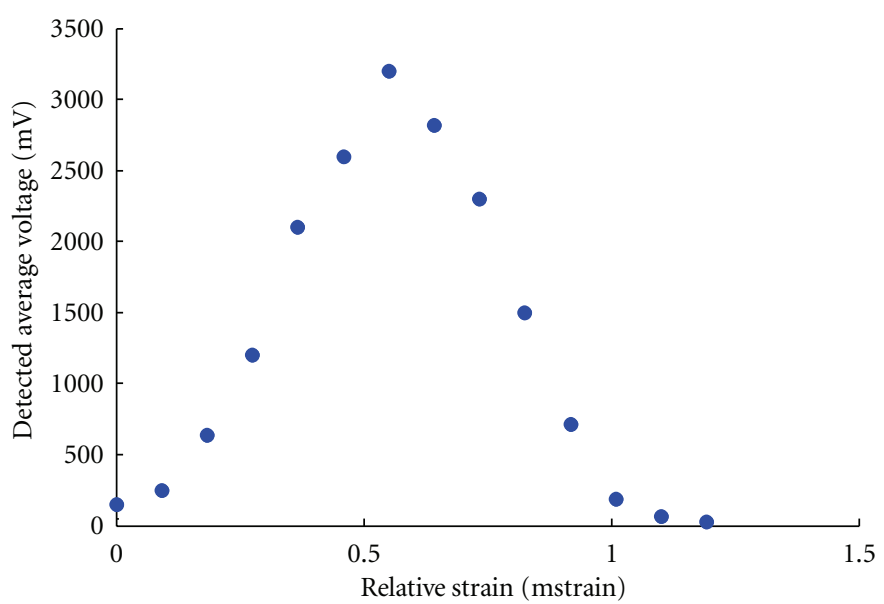

(b)

FIGURE 4: (a) Measured strain response for the four DBR sensors and (b) change in detected voltage as a function of applied strain for DBR .

\section{Detection of Acoustic Waves}

Acoustic waves are generated when cracks, deformations, and surface degradations are formed and their detection can be used for structural monitoring $[14,15]$. Several groups have investigated the use of all-fiber DBR lasers to detect acoustic waves; these implementations focus on exploiting the polarization dependence of refractive index changes related to acoustic pressure $[16,17]$. Acoustic waves are also associated with partial discharges (PDs), which occur in high-voltage systems when the insulation starts to degrade. It is extremely important to be able to detect the presence of PDs as early detection can help prevent critical degradation, which may lead to fires and hazards to neighboring equipment [18-21]. In this paper, we consider a simple approach based on detecting the time-varying changes in output power from a DBR laser that is subject to acoustic waves. Moreover, we show that since the DBR lasers are spatially independent, the effects of gain competition are minimized thereby eliminating crosstalk for multisensor operation.
To assess the capability of the DBR sensor to detect acoustic waves, we use a spark/electric arc discharge generator that produces acoustic waves that accompany the sparks, see Figure 5. The acoustic waves are then transmitted through a plastic or metallic surface/plate onto which the DBR is glued; the plate is located at a distance $H$ above the spark. The principle of operation is similar to the FBG hydrophone. Acoustic pressure arising from the acoustic waves can modulate the reflection spectrum of the FBG via the photoelastic effect and possible induced birefringence. However, the wavelength shifts are typically very small; on the other hand, when a pair of FBGs are incorporated into a laser cavity (e.g., the DBR laser), the small modulation on the reflection spectrum is enhanced owing to the feedback (lasing) process. Thus, the acoustic pressure modulates the output power and significantly narrower linewidth of the lasing signal (the linewidth is narrower than the FBG reflection bandwidth), resulting in a greater detection sensitivity [16].

The sparks are induced between two electrodes separated by a distance $L$ (typically less than $1 \mathrm{~cm}$ ). The acoustic waves 


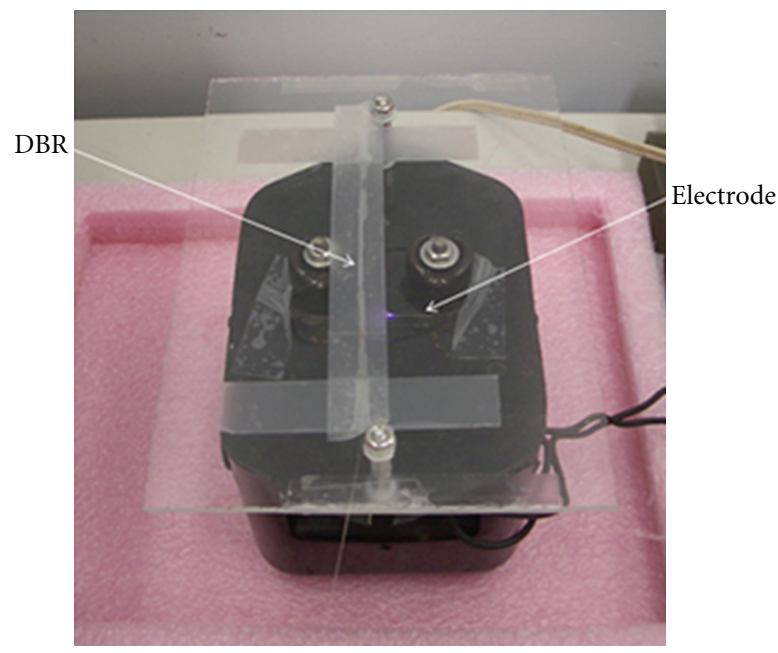

FIgURE 5: Photograph of setup for testing the all-fiber DBR sensor for detecting acoustic waves.

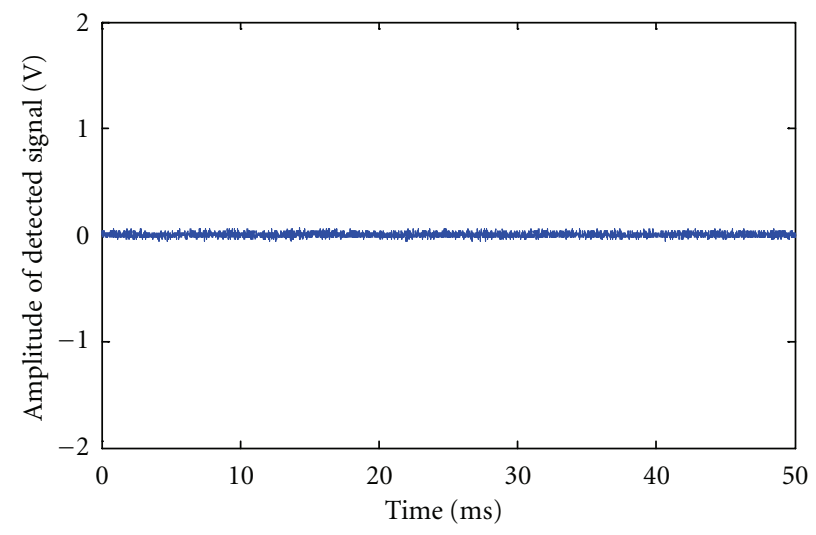

(a)

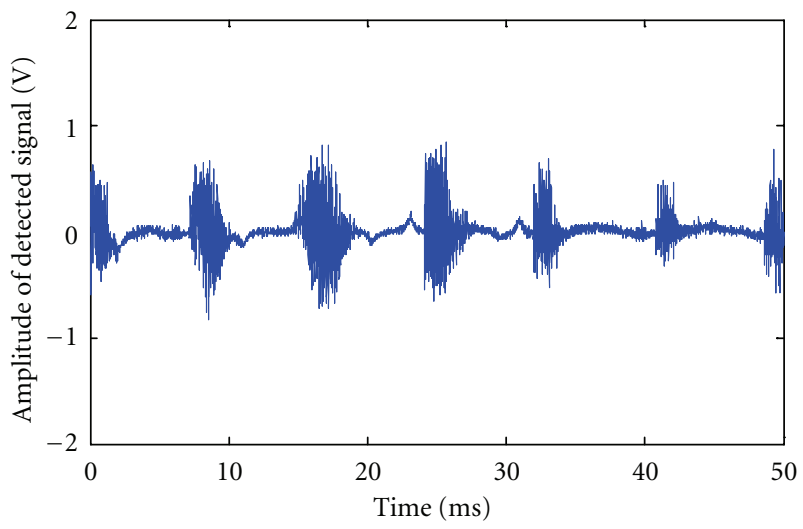

(b)

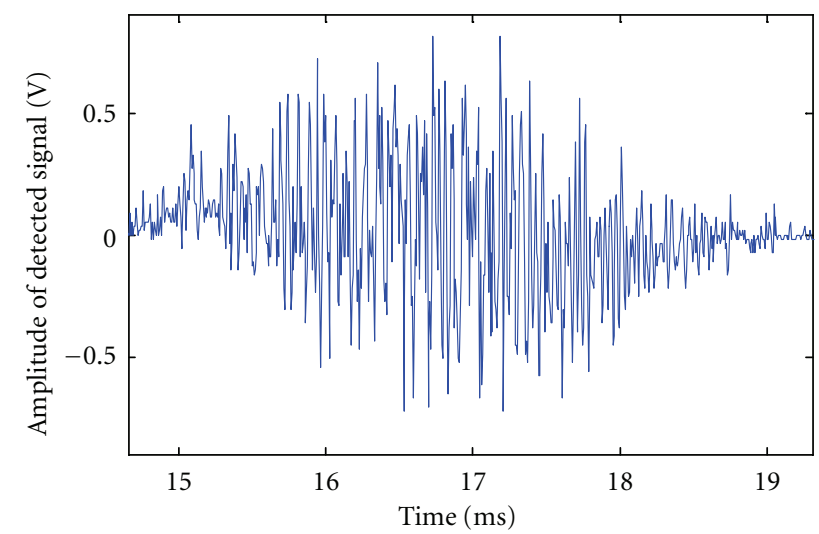

(c)

FIGURE 6: Detected signal from the DBR sensor (placed on the plastic plate) when (a) there is no spark and (b) a spark is generated. (c) Zoom of the third period over which the signal reflected from the DBR changes with time for the signal in (b).

distort the entire laser cavity and cause a fluctuation in the output power. We use a $200 \mathrm{kHz}$ photodetector to convert the optical signal from the DBR sensor to an electrical signal measured in time with an oscilloscope. Note that for a single sensor, there is no need to use wavelength-to-power mapping to detect the changes in power. However, the setup illustrated in Figure 2 can be used to detect the individual response of wavelength-multiplexed DBR sensors.

Figure 6 shows the detected signal when (a) no spark is generated and (b) a periodic spark is generated continuously 


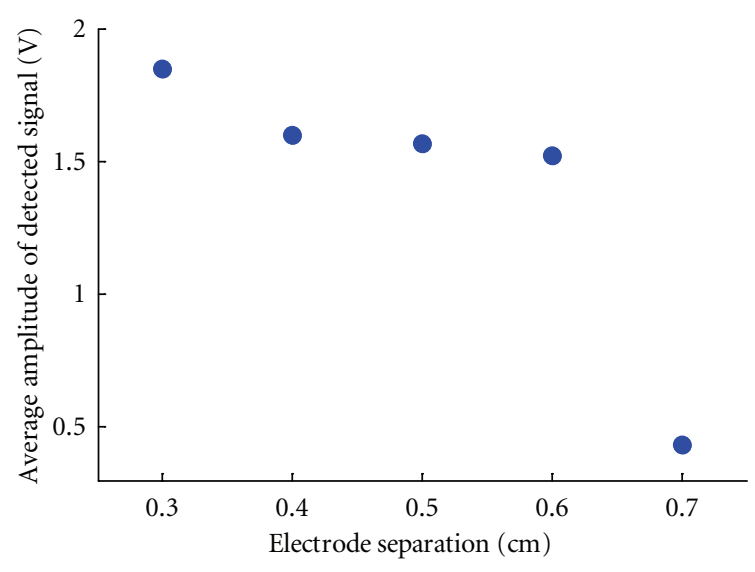

(a)

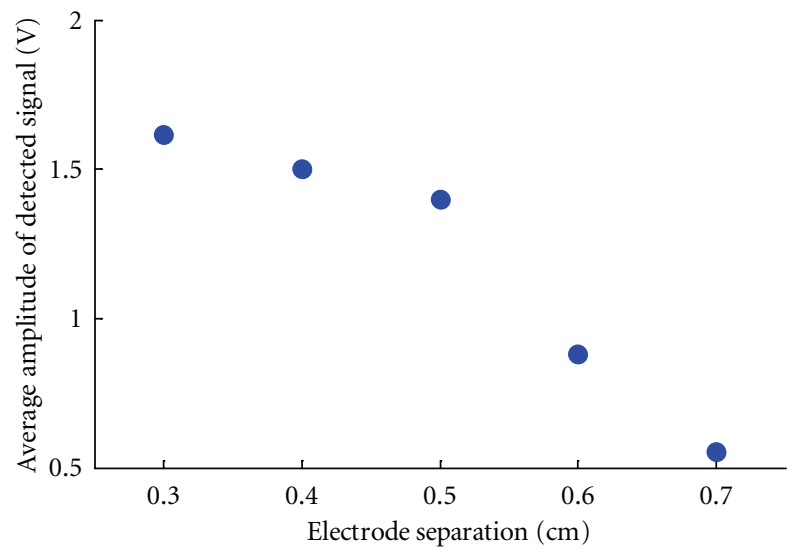

(b)

FIGURE 7: (a) Effect of increasing the electrode separation $L$ on the detected signal when the DBR is placed on (a) a plastic plate and (b) a metal plate. $H$ is fixed at $3.5 \mathrm{~cm}$.

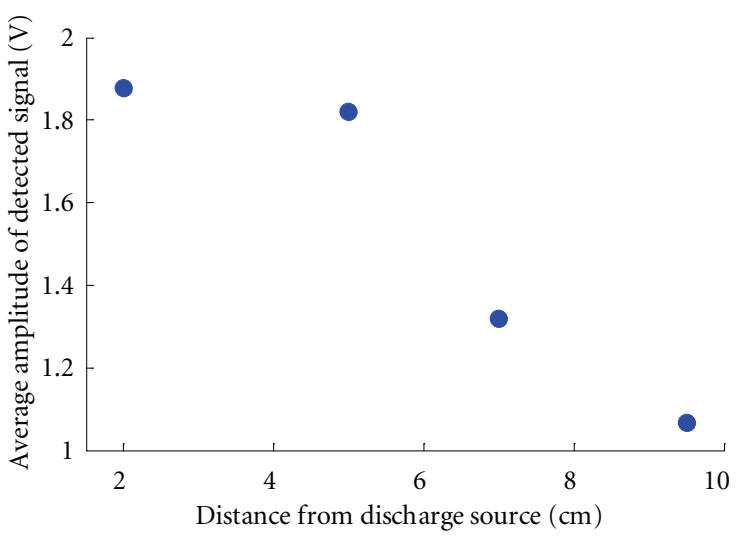

(a)

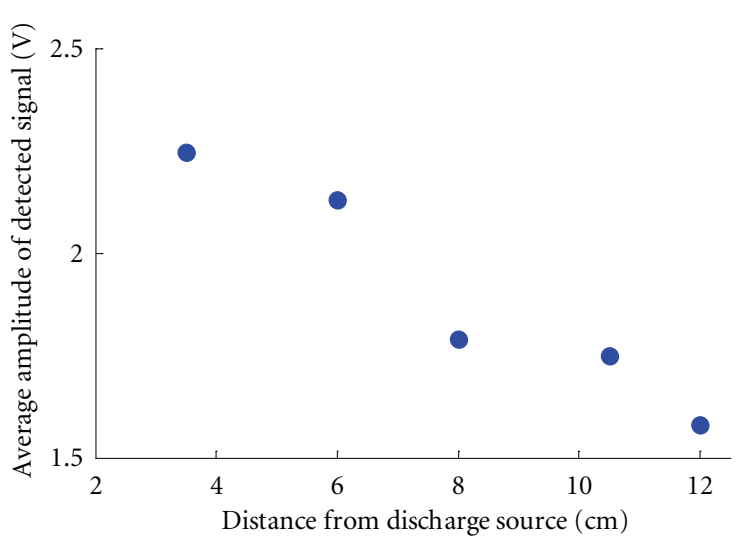

(b)

FIGURE 8: Effect of changing the distance $H$ between the spark and detection surface on the detected signal when the DBR is placed on (a) a plastic plate and (b) a metal plate. $L$ is fixed at $0.3 \mathrm{~cm}$.

beneath the plate. As observed, the latter experiences a periodic change in amplitude, showing that the DBR sensor is capable of capturing the acoustic wave emitted by the spark. Figure 6(c) illustrates a zoom of the time-varying signal associated with the acoustic wave.

We then characterize further the performance of the DBR sensor for detecting acoustic waves. First, we keep the distance $H$ of the metal or plastic plate above the location of the spark constant $(H=3.5 \mathrm{~cm})$ and vary the electrode separation $L$. The results are summarized in Figure 7: since increasing $L$ causes a decrease in the strength of the spark, the average maximum peak-to-peak amplitude of the detected signal also decreases. Second, we keep the electrode separation constant $(L=0.3 \mathrm{~cm})$ while varying $H$. As the plates are moved farther from the spark, we expect the detected signal to become weaker, and indeed, this is the case as shown in Figure 8.
Next, we investigate a wavelength multiplexed configuration (see Figure 9) to assess the crosstalk performance. We use two separate DBR sensors, one of which is subject to the acoustic waves arising from the sparks $\left(\mathrm{DBR}_{1}\right)$ and the other is isolated from the acoustic waves $\left(\mathrm{DBR}_{2}\right)$. As observed in Figure 9(a), when $\mathrm{DBR}_{1}$ is subject to the acoustic waves generated by the spark, the corresponding detected signal exhibits a periodic voltage variation. On the other hand, as shown in Figure 9(b), there is no variation in detected signal from $\mathrm{DBR}_{2}$. Figure 10 shows the RF spectra corresponding to the detected signals for $\mathrm{DBR}_{1}$ and $\mathrm{DBR}_{2}$. As illustrated, the spectral components of the signal corresponding to $\mathrm{DBR}_{1}$ are about $50 \mathrm{~dB}$ higher than those for $\mathrm{DBR}_{2}$. These measurements show that $\mathrm{DBR}_{2}$ is not affected by the changes imposed on $\mathrm{DBR}_{1}$; this demonstrates that crosstalk is negligible and that the sensors can be used in a wavelength-multiplexed configuration. Note that the use 


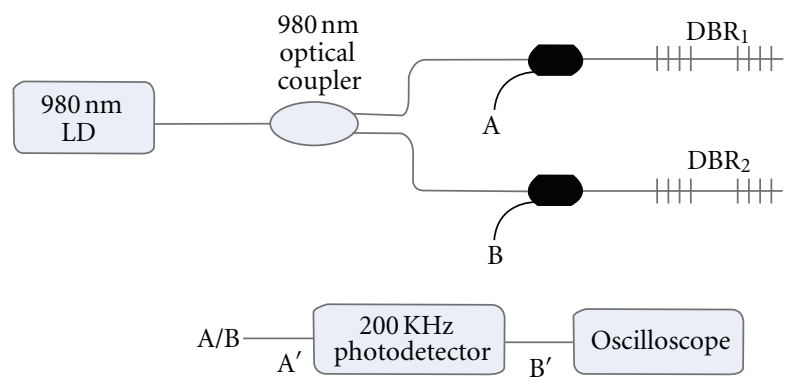

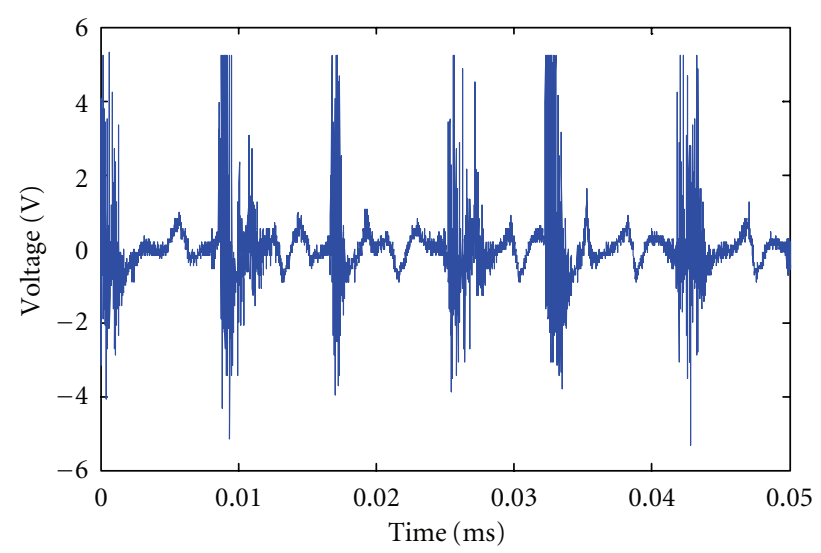

(a)

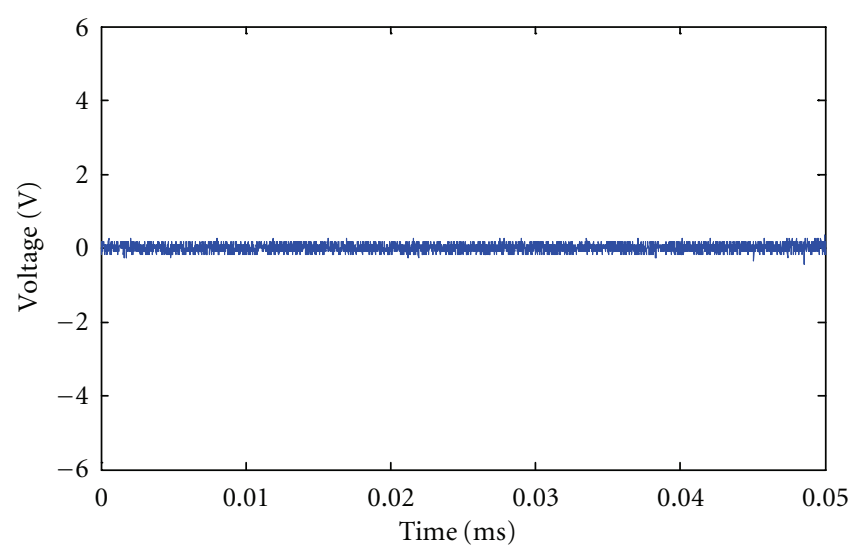

(b)

FIGURE 9: Experimental setup used to investigate crosstalk in a multi-DBR system (top). Detected signals from (a) $\mathrm{DBR}_{1}$ and (b) DBR 2 when $\mathrm{DBR}_{1}$ is subject to an acoustic wave arising from a spark.

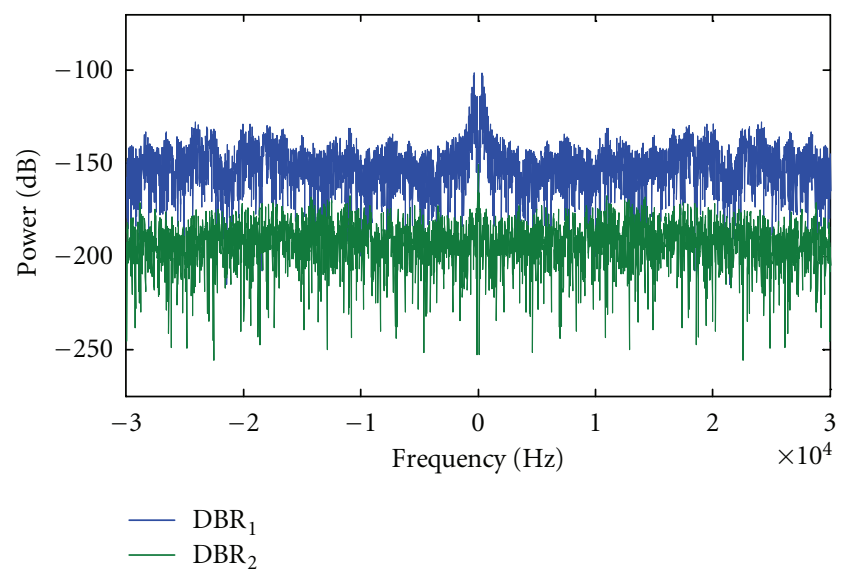

FIGURE 10: RF spectra for the detected signals from $\mathrm{DBR}_{1}$ and $\mathrm{DBR}_{2}$.

of different wavelengths allows for locating separate acoustic emissions if the DBR sensors are distributed spatially.

\section{Summary}

We have characterized the strain response of a DBR laser sensor system incorporating wavelength-to-power mapping. The system is demonstrated with 4 sensors; further scaling is possible, and the number of sensors that can be supported depends in large part on the available pump power. The measured strain sensitivities of the DBRs range from $0.84 \mathrm{~nm} / \mathrm{mstrain}$ to $0.88 \mathrm{~nm} / \mathrm{mstrain}$ and are similar to the typical values of FBGs ( $1 \mathrm{~nm} / \mathrm{mstrain})$. Moreover, we have demonstrated the possibility of using the DBR lasers for detecting acoustic waves transmitted through surfaces. Multiple sensors can be wavelength and spatially multiplexed to locate the spatial distribution of the acoustic waves. We showed that the response from each DBR sensor can be detected independently of the responses of the other sensors in the system, that is, the system is free from crosstalk. We believe that all-fiber DBR lasers can be used for a wide variety of fiber optic sensing applications.

\section{Acknowledgment}

This paper was supported in part by the Natural Sciences and Engineering Research Council of Canada via a collaborative research and development grant.

\section{References}

[1] M. Song, S. B. Lee, S. S. Choi, and B. Lee, "Simultaneous measurement of temperature and strain using two fiber Bragg gratings embedded in a glass tube," Optical Fiber Technology, vol. 3, no. 2, pp. 194-196, 1997.

[2] G. Chen, L. Liu, H. Jia, J. Yu, L. Xu, and W. Wang, "Simultaneous pressure and temperature measurement using Hi-Bi fiber Bragg gratings," Optics Communications, vol. 228, no. 1-3, pp. 99-105, 2003. 
[3] B. Lee and Y. Jeong, "Interrogation techniques for fiber grating sensors and the theory of fiber gratings," in Fiber Optic Sensors, F. T. S. Yu and S. Yin, Eds., pp. 295-382, Marcel Dekker, New York, NY, USA, 2002.

[4] R. W. Fallon, L. Zhang, A. Gloag, and I. Bennion, "Identical broadband chirped grating interrogation technique for temperature and strain sensing," Electronics Letters, vol. 33, no. 8, pp. 705-707, 1997.

[5] R. Huang, Y. Zhou, H. Cai, R. Qu, and Z. Fang, "A fiber Bragg grating with triangular spectrum as wavelength readout in sensor systems," Optics Communications, vol. 229, no. 1-6, pp. 197-201, 2004.

[6] Y. Sano and T. Yoshino, "Fast optical wavelength interrogator employing arrayed waveguide grating for distributed fiber Bragg grating sensors," Journal of Lightwave Technology, vol. 21, no. 1, pp. 132-139, 2003.

[7] G. Z. Xiao, P. Zhao, F. Sun, Z. Lu, and Z. Zhang, "Arrayedwaveguide-grating-based interrogator for wavelength-modulated multi-fiber-optic sensor applications," IEEE Photonics Technology Letters, vol. 17, no. 8, pp. 1710-1712, 2005.

[8] P. Niewczas, A. J. Willshire, L. Dziuda, and J. R. McDonald, "Performance analysis of the fiber Bragg grating interrogation system based on an arrayed waveguide grating," IEEE Transactions on Instrumentation and Measurement, vol. 53, no. 4, pp. 1192-1196, 2004.

[9] H. Su and X. G. Huang, "A novel fiber Bragg grating interrogating sensor system based on AWG demultiplexing," Optics Communications, vol. 275, no. 1, pp. 196-200, 2007.

[10] F. G. Sun, G. Z. Xiao, Z. Y. Zhang, and Z. G. Lu, "Modeling of arrayed waveguide grating for wavelength interrogation application," Optics Communications, vol. 271, no. 1, pp. 105$108,2007$.

[11] M. I. Comanici and L. R. Chen, "Improved arrayed waveguide grating-based interrogation system for fiber bragg grating sensors," in Proceedings of the 14th OptoElectronics and Communications Conference (OECC '09), Hong Kong, July 2009, Paper Q3.

[12] M.-I. Comanici, L. R. Chen, and P. Kung, "Measurement of dynamic strain using fiber Bragg grating-based laser sensor system," in Proceedings of the ICO International Conference on Information Photonics (IP '11), pp. 1-2, Ottawa, Ontario, Canada, May 2011.

[13] Y. Zhao and Y. Liao, "Discrimination methods and demodulation techniques for fiber Bragg grating sensors," Optics and Lasers in Engineering, vol. 41, no. 1, pp. 1-18, 2004.

[14] G. Wild and S. Hinckley, "Acousto-ultrasonic optical fiber sensors: overview and state-of-the-art," IEEE Sensors Journal, vol. 8, no. 7, pp. 1184-1193, 2008.

[15] C. B. Scruby, "An introduction to acoustic emission," Journal of Physics E, vol. 20, no. 8, pp. 946-953, 1987.

[16] B. O. Guan, H. Y. Tam, S. T. Lau, and H. L. W. Chan, "Ultrasonic hydrophone based on distributed Bragg reflector fiber laser," IEEE Photonics Technology Letters, vol. 17, no. 1, pp. 169-171, 2005.

[17] L. Y. Shao, S. T. Lau, X. Dong et al., "High-frequency ultrasonic hydrophone based on a cladding-etched DBR fiber laser," IEEE Photonics Technology Letters, vol. 20, no. 8, pp. 548-550, 2008.

[18] J. Deng, H. Xiao, W. Huo et al., "Optical fiber sensor-based detection of partial discharges in power transformers," Optics and Laser Technology, vol. 33, no. 5, pp. 305-311, 2001.

[19] G. C. Stone, V. Warren, H. G. Sedding, and W. McDermid, "Advances in interpreting partial discharge test results from motor and generator stator windings," in Proceedings of the
International Council of Large Electric Systems (CIGRE '02), 2002.

[20] G. C. Stone, E. A. Boulter, I. Culbert, and H. Dhirani, Electrical Insulation for Rotating Machines, Wiley Inter-Science, Danvers, Mass, USA, 2004.

[21] S. E. U. Lima, O. Frazão, R. G. Farias et al., "Mandrelbased fiber-optic sensors for acoustic detection of partial discharges-a proof of concept," IEEE Transactions on Power Delivery, vol. 25, no. 4, pp. 2526-2534, 2010. 

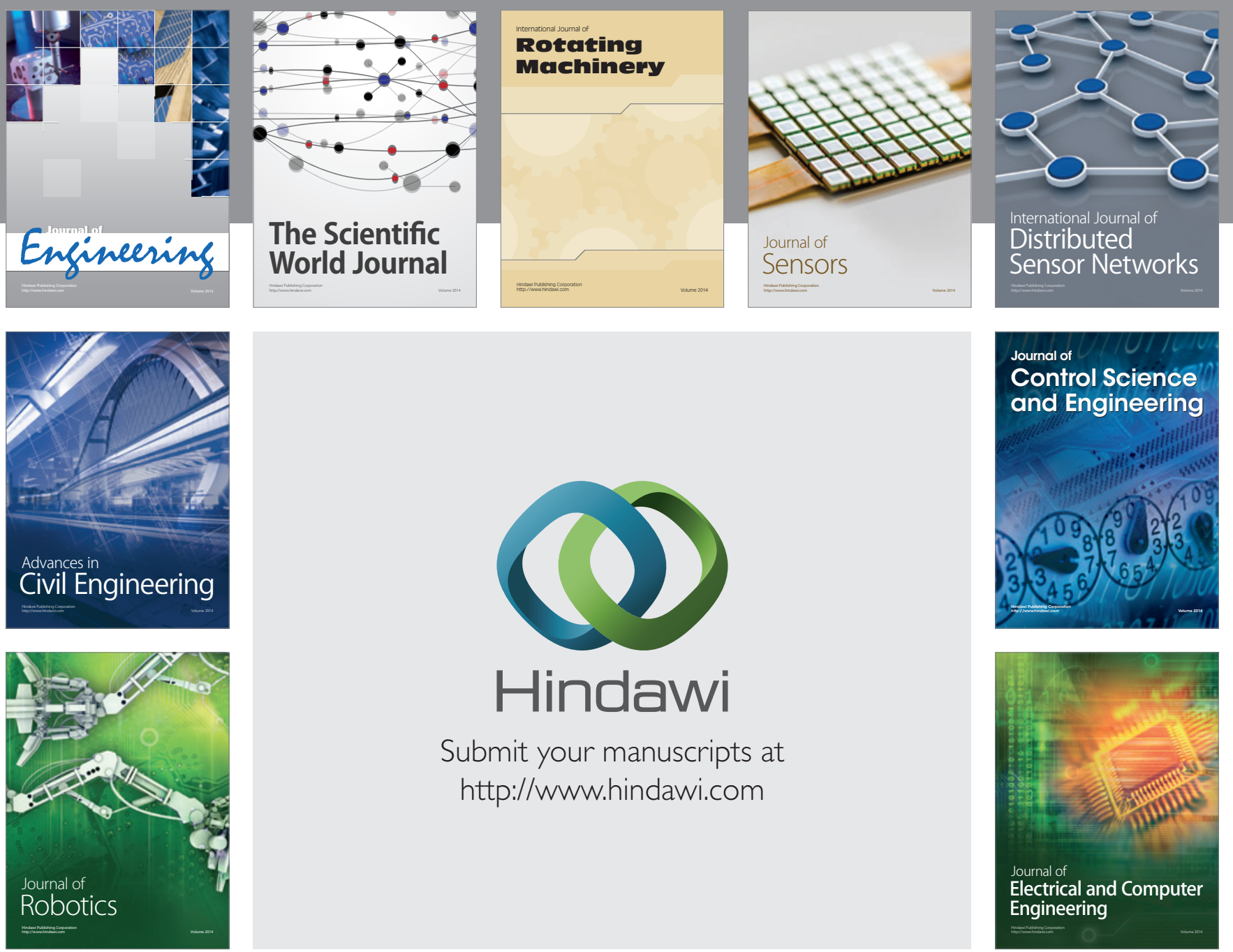

Submit your manuscripts at

http://www.hindawi.com
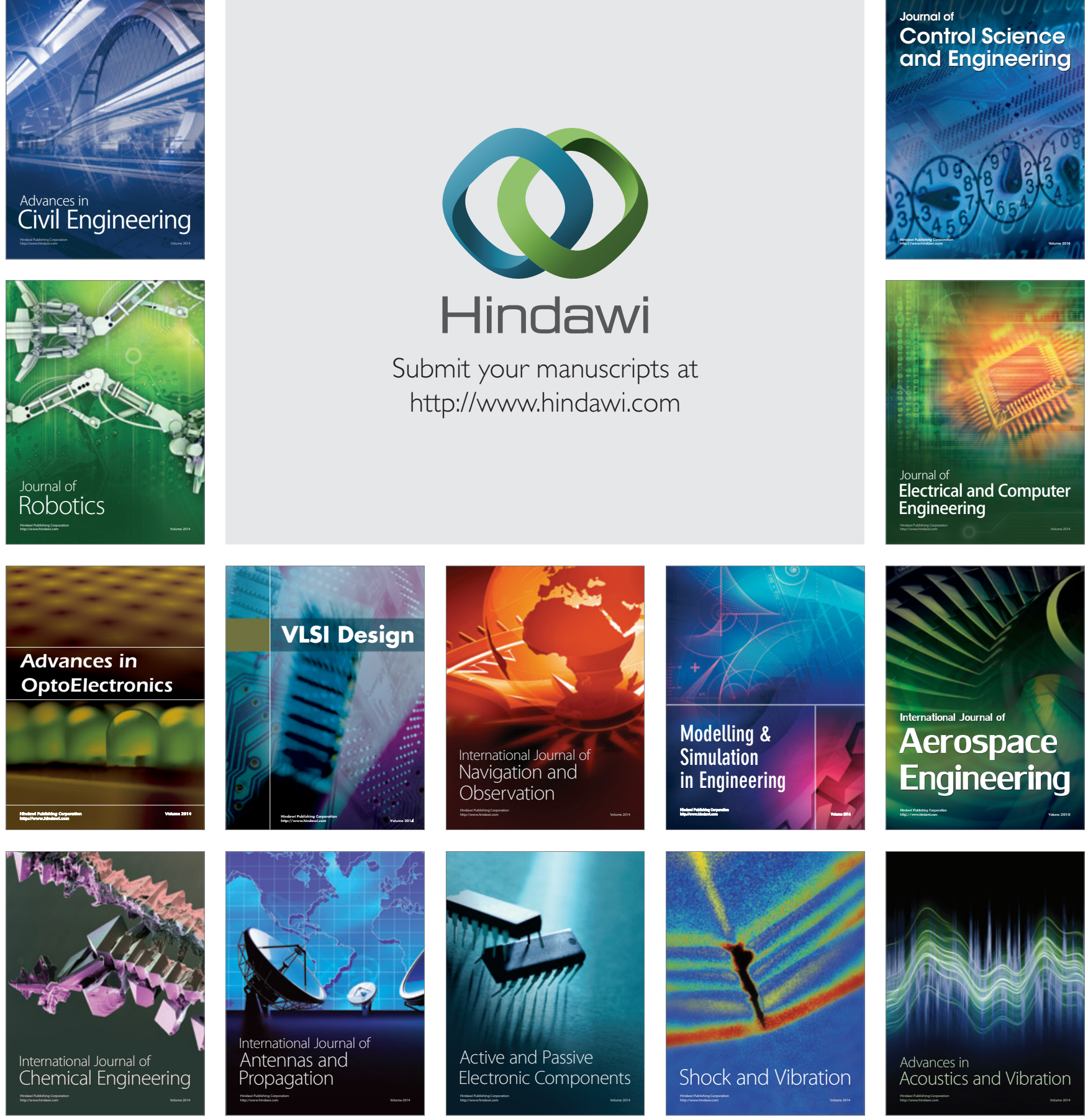